\title{
WATER USE EFFICIENCY OF DIFFERENT SUNFLOWER GENOTYPES UNDER DEFICIT IRRIGATION IN A SEMI-ARID REGION
}

\author{
MAHMOOD, H. N. ${ }^{*}-$ TOWFIQ, S. I. ${ }^{1}-$ RASHID, K. A. ${ }^{2}$ \\ ${ }^{1}$ Crop Science Department, College of Agricultural Sciences, University of Sulaimani, \\ Sulaimani-Kurdistan Region, Iraq \\ ${ }^{2}$ Soil and Water Science Department, College of Agricultural Sciences, University of Sulaimani \\ Sulaimani-Kurdistan Region, Iraq \\ *Corresponding author \\ e-mail: hekmat.mahmood@univsul.edu.iq; phone: +964-750-126-9838
}

(Received $30^{\text {th }}$ Oct 2018; accepted $11^{\text {th }}$ Jan 2019)

\begin{abstract}
The factorial experiment was set up in a randomized complete block design as a split-plot arrangement with three replications. Irrigation as main-plot consisted of four levels: stopping of irrigation after 60, 75, and 90 days from planting, and full irrigation. The sub-plot encompassed three sunflower genotypes (Barolo RO, Velko, and Local) used under a semiarid condition in Sulaimani, Kurdistan Region of Iraq. Velko genotype under full irrigation produced the highest seed yield of 5716.685, and $5190.545 \mathrm{~kg} \mathrm{ha}^{-1}$ at Kanipanka and Qlyasan locations respectively. Overall, 60 days of irrigation, and full irrigation showed the highest and lowest WUE or IWUE, respectively. All the genotypes offered a crop response factor of less than one at both locations indicating that the grown crop is more tolerant to water deficit, and recovers partially from stress. The result of this study concluded that the Barolo RO genotype has the highest performance under deficit irrigation. Therefore, this genotype is the most proper one for the study area as a part of drought-prone environments. Furthermore, the results obtained from this study indicated that the early stage of growth was more effective to increase the seed yield of sunflowers than irrigation in the middle and later stages.
\end{abstract}

Keywords: sunflower water productivity, seed yield, deficit irrigation, and yield response factor

\section{Introduction}

An estimated $70 \%$ of the world fresh water is used for agricultural productions, and this percentage could reach above $90 \%$ in some developing countries (WWP, 2017). The application of efficient water management strategies is a key element to increase water productivity (Mancosu et al., 2015). Further, water availability is one of the primary factors in sunflower seed production by irrigation depths (Gomes et al., 2012). The applied irrigation depths must be adequately quantified; otherwise, they may negatively affect the crop through water deficit or excess. Water deficit compromises photosynthesis, stomatal conductance, and transpiration, reducing plant biomass (Duarte et al., 2012). Water is decreasing day by day due to unavailability of natural rainfall, excessive withdrawal of groundwater, population growth, and increased use of irrigation water (Vörösmarty et al., 2000; Rosegrant et al., 2002; Rockström et al., 2009; Sarkar and Ali, 2009; Asraf et al., 2015). To deal with this situation, the lowest water usage with the highest yield has to be utilized in order to achieve maximum water productivity instead of highest yield, this method called, deficit irrigation and efficient use of water. Also, this technique can save irrigation cost with a negligible yield reduction consequently net farm income increase (Ali et al., 2007). This method shows a quantitative relationship between relative evaporation deficit and relative yield 
decrease. Therefore, to accept this theory, higher crop production and water productivity can be achieved with better water management planning (Ali, 2009). The sunflower plant is categorized as one of the low to medium drought-sensitive crop. Many researchers found that both quantity and distribution of water have a significant impact on achene and oil yield in sunflower (Fereres et al., 1986; Andrich et al., 1996; Krizmanic et al., 2003; Reddy et al., 2003; Iqbal et al., 2005). Deficit irrigation causes a yield reduction to some extent which depends on both the severity and timing of the water deficits (Orgaz et al., 1992). In water deficit condition (when it occurs during the life cycle) plant can achieve maximum water productivity (Ali, 2009). Ayas and Korukcu (2010) reported the $\mathrm{k}_{\mathrm{y}}$ factor of 0.909 in potato during growth period in Yenisehir, Bursa. Demir et al. (2006) estimated yield response factor $\mathrm{k}_{\mathrm{y}}$ of sunflower to deficit irrigation in a sub-humid climate (Bursa, Turkey). They found $\mathrm{k}_{\mathrm{y}}$ of 0.8382 for the total growth period of sunflower. However, by using furrow irrigation method (Karaata, 1991) found a $\mathrm{k}_{\mathrm{y}}$ value of 0.91 for the whole growing season and 0.83 for the vegetative + yielding stage. Hence, it is understandable that response factor differs from location to location depending on weather conditions, soil types, variety, crop, season and also for individual growth stage to the total growing season (Ali, 2009). Therefore, it is utmost essential, to consider location-specific response factor for efficient management of water. A hybrid yield was conditioned by its capacity to use the environmental variables efficiently in different phenophases (Gonzáles et al., 2013). Thus, the genetic potential of the sunflower hybrid was reduced by the action of the growth factors, either environmental or technological ones. Agronomic practices in addition to high yielding varieties are the two most important items for higher productivity of the sunflower crop (Beg et al., 2007). Productivity per unit area of sunflower was determined by many factors, including plant population and variety (Ibrahim, 2012). The objective of this study was to determine the effect of different deficit irrigation levels on the yield, irrigation water use efficiency (IWUE), water use efficiency (WUE), and yield response factor $\mathrm{k}_{\mathrm{y}}$ of some sunflower genotypes (Helianthus annuus L.) in the semi-arid conditions of Sulaimani region.

\section{Material and methods}

\section{Study area and soil sampling}

This experiment was conducted during the summer season of 2016 between July and October at two locations surrounding Sulaimani city. The first location was the Kanipanka Agricultural Research Station (latitude: $35^{\circ} 22$ ' 22" N, Longitude: $045^{\circ} 43^{\prime}$ 22" E, altitude: 548 masL) in Sharazoor valley. While the second location was Qlyasan, the farm of Crop Science Department, College of Agricultural Sciences, the University of Sulaimani, located at (latitude: $35^{\circ} 34^{\prime} 17^{\prime \prime} \mathrm{N}$, Longitude: $045^{\circ} 22^{\prime} 00^{\prime}$ " E, altitude: 757 masL) "Garmin, GPSmap60 Cx." (Fig. 1). The total available water at these two locations is 151 and $147 \mathrm{~mm} \mathrm{~m}^{-1}$.

At each location of the experiment, a composite soil sample of about $5 \mathrm{~kg}$ was obtained by mixing subsamples from 6 sites using a shovel. Each composite soil sample was freed from plant roots and other debris. Collected soil samples were placed in the open to be air dried at room temperature for about a week, then gently crushed and sieved at $2 \mathrm{~mm}$ with a stainless-steel sieve to avoid any contamination and then stored for subsequent analyses. 
Each experiment was set up in a randomized complete block design (RCBD) as a split-plot factorial arrangement with three replications with the deficit and full irrigation treatments as the main plot. Four levels were used: $I_{1}$ irrigation treatment (stopping of irrigation after 60 days from planting), $\mathrm{I}_{2}$ irrigation treatment (stopping of irrigation after 75 days from planting), $I_{3}$ irrigation treatment (stopping of irrigation after 90 days from planting), and $\mathrm{I}_{4}$ nonstop irrigation treatment (full irrigation), while the sub-plot factors encompassed three sunflower genotypes (Barolo RO, Velko, and Local). These genotypes were selected because of different responses to water stress.

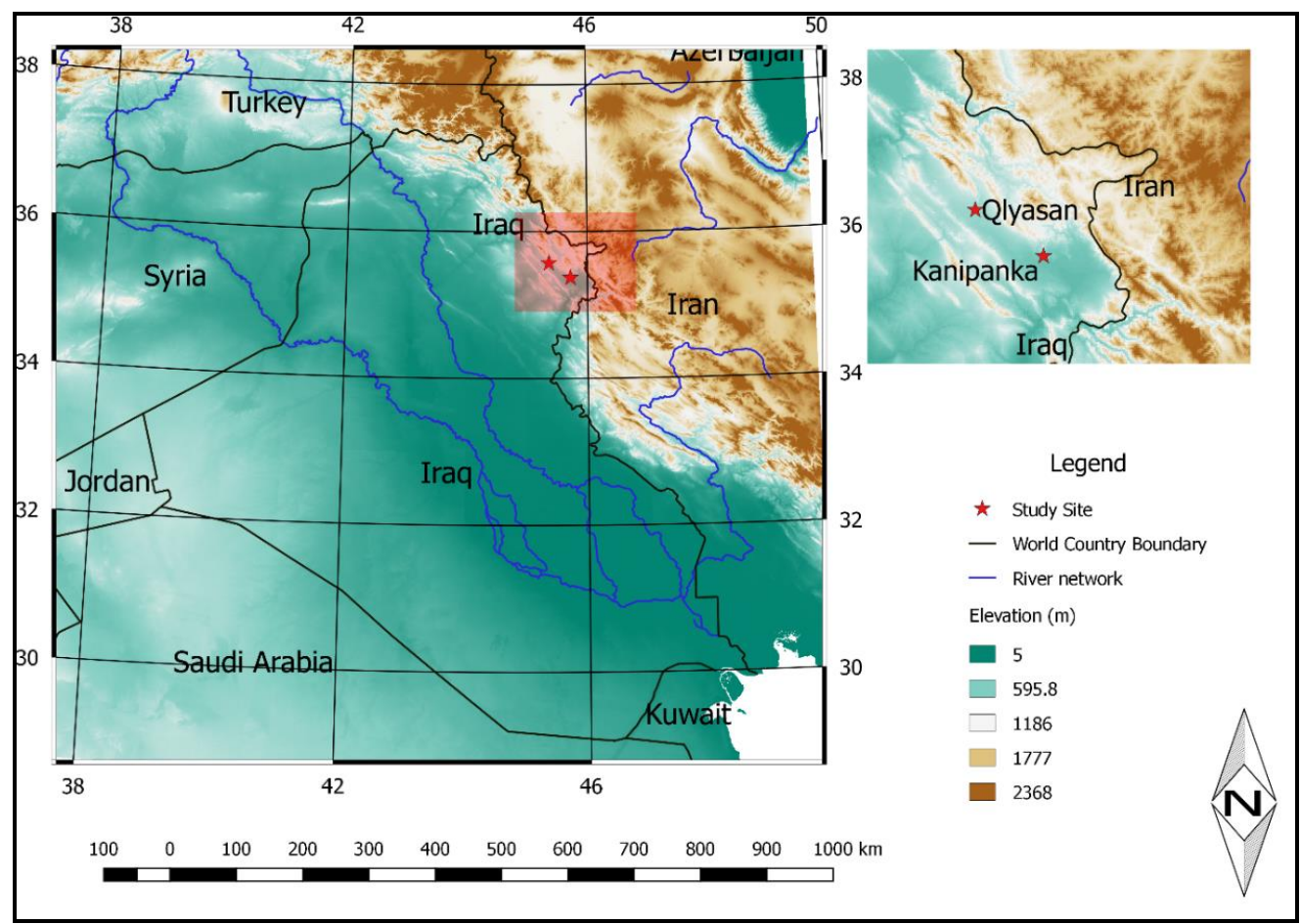

Figure 1. Map of the study site in Sulaimani-Kurdistan Region, Iraq

The sub-plots were $3 \mathrm{~m}$ by $1.8 \mathrm{~m}$ in size, and each consisted of three rows, spaced at $0.60 \mathrm{~m}$ with a plant distance of $0.30 \mathrm{~m}$. The seeds were planted during July $11^{\text {th }}$, and July $15^{\text {th }}$ at Kanipanka and Qlyasan location respectively. Three seeds hole ${ }^{-1}$ were placed at a depth of 2-4 cm. Two weeks after planting, the seedlings were thinned out to one plant hole ${ }^{-1}$. Urea, as a source of nitrogen fertilizer, was applied to all plots in two equal split doses before the second irrigation and prior to flowering at a rate of $43 \mathrm{~kg} \mathrm{ha}^{-}$ 1 as recommended. Hand weeding practiced as needed.

\section{Climate conditions of Sulaimani Governorate}

The climate of Sulaimani governorate is semi-arid environment: hot and dry in summer; cold and wet in winter. During July and August, the average temperature is between $39-43{ }^{\circ} \mathrm{C}$, and often reaching nearly $50{ }^{\circ} \mathrm{C}$. Autumn means high temperatures are $24-29^{\circ} \mathrm{C}$ in October, cooling slightly in November. Precipitation is limited to winter and spring months, and the overall average annual rainfall of $666.8 \mathrm{~mm}$ was at Sulaimani city in 2016 (Kurdistan Regional Government, 2018). An overview of experimental conditions is given in Table 1. 
Table 1. Some agrometeorological parameters at Kanipanka and Qlyasan locations

\begin{tabular}{|c|c|c|c|c|c|c|}
\hline \multirow{2}{*}{ Locations } & \multirow{2}{*}{ Month } & \multicolumn{2}{|c|}{ Air temperature $\left({ }^{\circ} \mathbf{C}\right)$} & \multirow{2}{*}{$\begin{array}{c}\text { Average } \\
\text { humidity }(\%)\end{array}$} & \multirow{2}{*}{$\begin{array}{l}\text { Average wind } \\
\text { speed }\left(\mathrm{m} \mathrm{s}^{-1}\right)\end{array}$} & \multirow{2}{*}{$\begin{array}{c}\text { Precipitation } \\
(\mathbf{m m})\end{array}$} \\
\hline & & Minimum & Maximum & & & \\
\hline \multirow{4}{*}{ Kanipanka } & July & 27.05 & 44.33 & 20.30 & 1.65 & 0 \\
\hline & August & 29.34 & 45.88 & 19.56 & 1.5 & 0 \\
\hline & September & 21.42 & 39.55 & 22.68 & 1.61 & 0 \\
\hline & October & 15.66 & 34.69 & 28.56 & 1.38 & 0 \\
\hline \multirow{4}{*}{ Qlyasan } & July & 25.84 & 43.46 & 22.9 & 1.77 & 0 \\
\hline & August & 27.49 & 45.36 & 20.35 & 1.6 & 0 \\
\hline & September & 19.95 & 38.26 & 26.17 & 1.65 & 0 \\
\hline & October & 14.95 & 32.01 & 29.42 & 1.44 & 0 \\
\hline
\end{tabular}

\section{Watering schedule and restrictions}

Irrigation timing method was based on an allowable root zone available water depletion (45\%) during full and no deficit irrigation (Allen et al., 1998). The amount of irrigation water was measured with water flow meter devices (SOTERA digital display meter) (Fig. 2). The soil water content was monitored gravimetrically (Lorenz and Maynard, 1980), using a small auger $5 \mathrm{~cm}$ in diameter. The net water requirement (crop consumptive use) was calculated from soil moisture. When the available soil moisture was depleted by $45 \%$, the soil moisture was brought to filed capacity. The sum of the crop consumptive use during the growing season was comparable well with that computed by Penman-Monteith equation

At each location, the matured plants from the central rows in each plot were harvested manually and then yield and yield components for each treatment at each replicate were determined. The yield data was taken at about $10 \%$ seed moisture level. The harvesting dates were on October $19^{\text {th }}, 2016$ for the deficit irrigation treatments, and October $24^{\text {th }}$ for the full irrigation treatment at Kanipanka location, while October $25^{\text {th }}$ were the harvesting dates for all the treatments at Qlyasan location respectively.

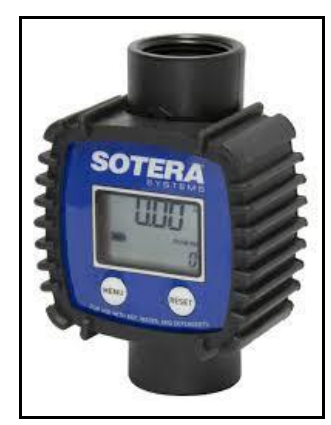

Figure 2. SOTERA digital display meter

The below-stated equation was used to estimate water use efficiency (WUE, Eq. 1), and irrigation water use efficiency (IWUE, Eq. 2), as the ratio of crop yield per unit of water applied (Kang et al., 2000):

$$
W U E=\frac{Y}{E T E}
$$




$$
\text { IWUE }=\frac{Y}{I}
$$

where:

$Y=$ The total sunflower seed yield $\left(\mathrm{kg} \mathrm{ha}^{-1}\right)$

$E T C=$ The seasonal evapotranspiration $\left(\mathrm{m}^{3} \mathrm{ha}^{-1}\right)$

$I=$ The total volume of applied irrigation water $\left(\mathrm{m}^{3} \mathrm{ha}^{-1}\right)$

While the irrigation application efficiencies for the locations under study based on average land slop and basic infiltration rate was calculated according to Karim and Karim (2001) and the results are revealed in Table 2.

Table 2. Irrigation application efficiencies for the locations under study based on average land slope and basic infiltration rate

\begin{tabular}{c|c|c|c}
\hline Locations & Average land slope $\mathbf{( \% )}$ & $\begin{array}{c}\text { Basic infiltration rate, } \\
\mathbf{I b}\left(\mathbf{m m h r}^{-1}\right)\end{array}$ & $\begin{array}{c}\text { Irrigation application } \\
\text { efficiency, Ea }\end{array}$ \\
\hline Kanipanka & Nearly level & 44 & 0.70 \\
Qlyasan & 1.16 & 80 & 0.65 \\
\hline
\end{tabular}

The crop response factor $K y$ is the relationship between relative yield decrease and relative evapotranspiration deficit which was determined by the procedure given by Doorenbos and Kassam (1979) (Eq. 3).

$$
\left(1-\frac{Y a}{Y m}\right)=K y\left(1-\frac{E T a}{E T \max }\right)
$$

where:

$Y a=$ Actual crop yield $\left(\mathrm{kg} \mathrm{ha}^{-1}\right)$

$Y m=$ Maximum crop yield $\left(\mathrm{kg} \mathrm{ha}^{-1}\right)$

$E t a=$ Actual evapotranspiration $(\mathrm{mm})$

$E T m=$ Maximum evapotranspiration $(\mathrm{mm})$

$K y=$ Yield response factor (dimensionless)

The $\mathrm{k}_{\mathrm{y}}$ factor for the entire season determined by linear regression, adjusted through the origin, between the reduction in relative yield and deficit of relative evapotranspiration.

The uniformity coefficient (UC, Eq. 4) for some selected parameters was determined according to Devitt et al. (1992):

$$
U C=1-(\text { standard deviation } / \text { mean })
$$

\section{Analytical methods and laboratory analysis}

The results of the studied soil parameters are shown in Table 3. Particle size fractionation and distribution were conducted by international pipette method as recommended by Black et al. (1965). EC and acidity ( $\mathrm{pH})$ of soil sample were measured in 1:10, soil to $\mathrm{H}_{2} \mathrm{O}$ ratio suspension according to Thomas (1996) by using these models of instruments; pH-meter (model WTW 330i/ Germany); EC-meter (model WTW 330i/Germany). The percent of organic carbon (o.m\%) in soil samples were determined by the Walkley-Black method (wet oxidation by potassium dichromate $\mathrm{K}_{2} \mathrm{Cr}_{2} \mathrm{O}_{7}$ and concentrated $\mathrm{H}_{2} \mathrm{SO}_{4}$ ) as described by Black et al. (1965). Then the content of organic 
matter $(\mathrm{OM})$ was calculated as follows: $\%$ Organic matter $=\%$ organic carbon $\times 1.724$ (factor). The percent of the total $\left(\mathrm{CaCO}_{3} \%\right)$ was determined by the acid-neutralization method according to the method 23c of U.S. Salinity Laboratory Staff, 1954 (Black et al., 1965).

Table 3. Some physicochemical properties of the soil samples for locations of the experiment

\begin{tabular}{|c|c|c|c|}
\hline \multirow{2}{*}{\multicolumn{2}{|c|}{ Physicochemical properties }} & \multicolumn{2}{|c|}{ Locations } \\
\hline & & \multirow{2}{*}{$\begin{array}{c}\text { Kanipanka } \\
36\end{array}$} & \multirow{2}{*}{$\begin{array}{c}\text { Qlyasan } \\
87\end{array}$} \\
\hline \multirow{4}{*}{$\begin{array}{l}\text { Particles size distribution } \\
\qquad\left(\mathrm{kg}^{-1}\right)\end{array}$} & Sand & & \\
\hline & Silt & 529 & 435 \\
\hline & Clay & 435 & 458 \\
\hline & Texture & $\mathrm{SiC}$ & $\mathrm{SiC}$ \\
\hline \multicolumn{2}{|l|}{$\mathrm{PH}$} & 7.70 & 7.59 \\
\hline \multicolumn{2}{|c|}{$\mathrm{ECe}\left(\right.$ micro siemens $\left.\mathrm{cm}^{-1}\right)$ or $\left(\mu \mathrm{S} \mathrm{cm}^{-1}\right)$} & 218 & 490 \\
\hline \multicolumn{2}{|c|}{ O.M. $\left(\mathrm{g} \mathrm{kg}^{-1}\right)$} & 14.8 & 22.4 \\
\hline \multicolumn{2}{|c|}{$\mathrm{CaCO} 3\left(\mathrm{~g} \mathrm{~kg}^{-1}\right)$} & 208.3 & 304.3 \\
\hline
\end{tabular}

\section{Statistical analysis}

Statistical analysis for all measured variable was performed using the XLSTAT software (XLSTAT, 2017). For direct comparison of treatments, least significant difference tests (LSD) at levels of 0.05 and 0.01 levels were used. For testing the main effects of deficit irrigation on sunflower genotypes in a semi-arid region, the data were subjected to analysis of variance (ANOVA).

\section{Results}

Table 4 shows the seed yields, for irrigation water use efficiency (IWUE) and water use efficiency (WUE) for all three genotypes of sunflower at Kanipanka and Qlyasan locations under different deficit irrigation treatments during the summer season of 2016.

As seen in Table 4, highly significant differences among genotypes were recorded (see Appendix). The highest value of seed yield recorded with the Velko genotype under $\mathrm{I}_{4}$ (full irrigation) at first location was $5716.685 \mathrm{~kg} \mathrm{ha}^{-1}$ which predominated all combinations significantly while the lowest value was $2915.600 \mathrm{~kg} \mathrm{ha}^{-1}$ recorded with the Local genotype under $I_{1}$ (stopping of irrigation after 60 days) at the same locations. The irrigation water use efficiency varied from as low as $4.511 \mathrm{~kg} \mathrm{ha}^{-1} \mathrm{~mm}^{-1}$ for the Local genotype under $\mathrm{I}_{3}$ (stopping of irrigation after 90 days) at the second location to as high as $9.382 \mathrm{~kg} \mathrm{ha}^{-1} \mathrm{~mm}^{-1}$ for the Velko genotype under $\mathrm{I}_{1}$ (stopping of irrigation after 60 days) at the same location, in which exceeded other combinations significantly. On the other hand, it was reported that the water use efficiency varied from a minimum of $6.754 \mathrm{~kg} \mathrm{ha}^{-1} \mathrm{~mm}^{-1}$ for the Barolo RO genotype under $\mathrm{I}_{4}$ (full irrigation) at the first location to a maximum of $14.437 \mathrm{~kg} \mathrm{ha}^{-1} \mathrm{~mm}^{-1}$ for the Velko genotype under $\mathrm{I}_{1}$ (stopping of irrigation after 60 days) at the second location.

Based on the average values of IWUE, and WUE for the two locations, the order of performance of the genotypes is as follows: Velko > Barolo RO > Local. Our study showed that the Velko and Local genotypes offered the highest and lowest performance respectively. 
Table 4. Seed yield, irrigation water use efficiency, and water use efficiency of three sunflower genotypes as influenced by different irrigation treatments at two locations within Sulaimani City

\begin{tabular}{|c|c|c|c|c|c|c|c|}
\hline \multirow{2}{*}{\multicolumn{2}{|c|}{$\begin{array}{l}\text { Sunflower genotypes and } \\
\text { irrigation treatments }\end{array}$}} & \multicolumn{3}{|c|}{ Total $x$} & \multirow{2}{*}{$\begin{array}{c}\text { Seed yield } \\
\left(\mathrm{kg} \mathrm{ha}^{-1}\right)\end{array}$} & \multirow{2}{*}{$\begin{array}{c}\text { Irrigation water } \\
\text { use efficiency } \\
\left(\mathrm{kg} \mathrm{ha}^{-1} \mathbf{~ m m}^{-1}\right)\end{array}$} & \multirow{2}{*}{$\begin{array}{c}\text { Water use } \\
\text { efficiency } \\
\left(\mathrm{kg} \mathrm{ha}^{-1} \mathbf{m m}^{-1}\right)\end{array}$} \\
\hline & & $(\mathbf{m m})$ & $\left(\mathbf{m}^{3} \mathbf{h} \mathbf{a}^{-1}\right)$ & $\mathbf{E T}_{\mathbf{a}}$ & & & \\
\hline \multicolumn{8}{|c|}{ Kanipanka location } \\
\hline \multirow{4}{*}{$\begin{array}{c}\text { Barolo } \\
\text { RO }\end{array}$} & $\begin{array}{c}\mathrm{I}_{1} \text { stopping of } \\
\text { irrigation after } 60 \\
\text { days }\end{array}$ & 455.37 & 4553.7 & 3187.59 & $2977.020 \mathrm{e}$ & $6.538 \mathrm{~cd}$ & $9.339 \mathrm{~cd}$ \\
\hline & $\begin{array}{c}\mathrm{I}_{2} \text { stopping of } \\
\text { irrigation after } 75 \\
\text { days }\end{array}$ & 632.77 & 6327.77 & 4429.439 & $3269.690 \mathrm{de}$ & $5.167 \mathrm{e}$ & $7.382 \mathrm{e}$ \\
\hline & \begin{tabular}{|c|}
$\mathrm{I}_{3}$ Stopping of \\
irrigation after 90 \\
days
\end{tabular} & 713.7 & 7137.03 & 4995.921 & $3541.455 \mathrm{~d}$ & $4.962 \mathrm{e}$ & $7.089 \mathrm{e}$ \\
\hline & $\mathrm{I}_{4}$ Full irrigation & 761.3 & 7612.96 & 5329.072 & $3599.175 \mathrm{~d}$ & $4.728 \mathrm{e}$ & $6.754 \mathrm{e}$ \\
\hline \multirow{4}{*}{ Velko } & \begin{tabular}{|c|}
$\mathrm{I}_{1}$ Stopping of \\
Irrigation after 60 \\
Days
\end{tabular} & 455.37 & 4553.7 & 3187.59 & $4168.790 \mathrm{c}$ & $9.155 \mathrm{a}$ & $13.078 \mathrm{a}$ \\
\hline & \begin{tabular}{|c|}
$\mathrm{I}_{2}$ Stopping of \\
irrigation after 75 \\
days
\end{tabular} & 632.77 & 6327.77 & 4429.439 & $4178.780 \mathrm{c}$ & $6.604 \mathrm{~cd}$ & $9.434 \mathrm{~cd}$ \\
\hline & \begin{tabular}{|c|}
$\mathrm{I}_{3}$ Stopping of \\
irrigation after 90 \\
days
\end{tabular} & 713.7 & 7137.03 & 4995.921 & $5149.475 \mathrm{~b}$ & $7.215 \mathrm{bc}$ & $10.307 \mathrm{bc}$ \\
\hline & $\mathrm{I}_{4}$ Full irrigation & 761.3 & 7612.96 & 5329.072 & $5716.685 \mathrm{a}$ & $7.509 \mathrm{~b}$ & $10.727 \mathrm{~b}$ \\
\hline \multirow{4}{*}{ Local } & $\begin{array}{c}\mathrm{I}_{1} \text { Stopping of } \\
\text { irrigation after } 60 \\
\text { days }\end{array}$ & 455.37 & 4553.7 & 3187.59 & $2915.600 \mathrm{e}$ & $6.403 \mathrm{~d}$ & $9.147 \mathrm{~d}$ \\
\hline & \begin{tabular}{|c|}
$\mathrm{I}_{2}$ Stopping of \\
irrigation after 75 \\
days
\end{tabular} & 632.77 & 6327.77 & 4429.439 & $3389.94 \mathrm{~d}$ & $5.357 \mathrm{e}$ & $7.653 \mathrm{e}$ \\
\hline & \begin{tabular}{|c|}
$\mathrm{I}_{3}$ Stopping of \\
irrigation after 90 \\
days
\end{tabular} & 713.7 & 7137.03 & 4995.921 & $3491.690 \mathrm{~d}$ & $4.892 \mathrm{e}$ & $6.989 \mathrm{e}$ \\
\hline & $\mathrm{I}_{4}$ Full irrigation & 761.3 & 7612.96 & 5329.072 & $3670.215 \mathrm{~d}$ & $4.821 \mathrm{e}$ & $6.887 \mathrm{e}$ \\
\hline \multicolumn{5}{|c|}{$\mathrm{LSD}_{.05}$} & 404.034 & 0.689 & 0.984 \\
\hline \multicolumn{8}{|c|}{ Qlyasan location } \\
\hline \multirow{4}{*}{$\begin{array}{c}\text { Barolo } \\
\text { RO }\end{array}$} & \begin{tabular}{|c|}
$\mathrm{I}_{1}$ Stopping of \\
irrigation after 60 \\
days
\end{tabular} & 452.04 & 4520.37 & 2938.241 & $3644.685 \mathrm{~d}$ & $8.063 \mathrm{~b}$ & $12.404 \mathrm{~b}$ \\
\hline & \begin{tabular}{|c|}
$\mathrm{I}_{2}$ Stopping of \\
irrigation after 75 \\
days
\end{tabular} & 583.33 & 5833.33 & 3791.665 & $3836.160 \mathrm{~d}$ & $6.576 \mathrm{e}$ & $10.117 \mathrm{e}$ \\
\hline & \begin{tabular}{|c|}
$\mathrm{I}_{3}$ Stopping of \\
irrigation after 90 \\
days
\end{tabular} & 706.3 & 7062.96 & 4590.924 & $4116.620 \mathrm{c}$ & $5.828 \mathrm{f}$ & $8.967 \mathrm{f}$ \\
\hline & $\mathrm{I}_{4}$ Full irrigation & 754.26 & 7542.59 & 4902.684 & $4192.285 \mathrm{c}$ & $5.558 \mathrm{f}$ & $8.551 \mathrm{f}$ \\
\hline
\end{tabular}




\begin{tabular}{|c|c|c|c|c|c|c|c|}
\hline \multirow{4}{*}{ Velko } & $\begin{array}{c}\mathrm{I}_{1} \text { Stopping of } \\
\text { irrigation after } 60 \\
\text { days }\end{array}$ & 452.04 & 4520.37 & 2938.241 & $4242.05 \mathrm{c}$ & $9.382 \mathrm{a}$ & $14.437 \mathrm{a}$ \\
\hline & $\begin{array}{c}\mathrm{I}_{2} \text { Stopping of } \\
\text { irrigation after } 75 \\
\text { days }\end{array}$ & 583.33 & 5833.33 & 3791.665 & $4817.030 \mathrm{~b}$ & $8.258 \mathrm{~b}$ & $12.704 \mathrm{~b}$ \\
\hline & $\begin{array}{c}\mathrm{I}_{3} \text { Stopping of } \\
\text { irrigation after } 90 \\
\text { days }\end{array}$ & 706.3 & 7062.96 & 4590.924 & $4910.825 \mathrm{~b}$ & $6.953 \mathrm{~cd}$ & $10.697 \mathrm{~cd}$ \\
\hline & $\mathrm{I}_{4}$ Full Irrigation & 754.26 & 7542.59 & 4902.684 & $5190.545 \mathrm{a}$ & $6.882 \mathrm{de}$ & $10.587 \mathrm{de}$ \\
\hline \multirow{4}{*}{ Local } & $\begin{array}{c}\mathrm{I}_{1} \text { Stopping of } \\
\text { irrigation after } 60 \\
\text { days } \\
\end{array}$ & 452.04 & 4520.37 & 2938.241 & $3283.01 \mathrm{e}$ & $7.263 \mathrm{c}$ & $11.173 \mathrm{c}$ \\
\hline & \begin{tabular}{|c|}
$\mathrm{I}_{2}$ Stopping of \\
irrigation after 75 \\
days
\end{tabular} & 583.33 & 5833.33 & 3791.665 & $3214.19 \mathrm{e}$ & $5.510 \mathrm{f}$ & $8.477 \mathrm{f}$ \\
\hline & \begin{tabular}{|c|}
$\mathrm{I}_{3}$ Stopping of \\
irrigation after 90 \\
days \\
\end{tabular} & 706.3 & 7062.96 & 4590.924 & $3186.440 \mathrm{e}$ & $4.511 \mathrm{~h}$ & $6.941 \mathrm{~h}$ \\
\hline & $\mathrm{I}_{4}$ Full irrigation & 754.26 & 7542.59 & 4902.684 & $3738.295 \mathrm{~d}$ & $4.956 \mathrm{~g}$ & $7.625 \mathrm{~g}$ \\
\hline \multicolumn{5}{|c|}{$\operatorname{LSD}_{.05}$} & 253.587 & 0.375 & 0.577 \\
\hline
\end{tabular}

As expected the percent yield reduction decreased progressively with an increase in the amount of applied water. Further, the Velko genotype offered the maximum percent of the reduction in yield $(27.08 \%)$ under $\mathrm{I}_{1}$ (stopping of irrigation after 60 days) at the first location. Table 5 revealed that water stress imposed at the later stage of growth influence yield the least, and offered water saving of about $40 \%$ compared with the full irrigation treatment. The percent of the reduction under limited irrigation was less than $20 \%$ in most cases (Table 5).

Table 5. Percent of yield reduction and water saving under limited irrigation

\begin{tabular}{|c|c|c|c|c|c|}
\hline \multicolumn{2}{|c|}{$\begin{array}{l}\text { Sunflower genotypes and } \\
\text { irrigation treatments }\end{array}$} & $\begin{array}{c}\text { Total applied } \\
\text { water }(\mathbf{m m})\end{array}$ & $\begin{array}{c}\text { Seed yield } \\
\left(\mathrm{Kg} \mathrm{ha}^{-1}\right)\end{array}$ & \begin{tabular}{|c|}
$\begin{array}{c}\text { Yield reduction } \\
(\%)\end{array}$ \\
\end{tabular} & $\begin{array}{c}\text { Water saving } \\
(\%)\end{array}$ \\
\hline \multicolumn{6}{|c|}{ Kanipanka location } \\
\hline \multirow{4}{*}{$\begin{array}{l}\text { Barolo } \\
\text { RO }\end{array}$} & $\begin{array}{c}\mathrm{I}_{1} \text { Stopping of } \\
\text { irrigation after } 60 \text { days }\end{array}$ & 455.370 & $2977.020 \mathrm{e}$ & 17.29 & 40.19 \\
\hline & \begin{tabular}{|c|}
$\mathrm{I}_{2}$ Stopping of \\
irrigation after 75 days
\end{tabular} & 632.770 & $3269.690 \mathrm{de}$ & 9.15 & 16.88 \\
\hline & \begin{tabular}{|c|}
$\mathrm{I}_{3}$ Stopping of \\
irrigation after 90 days
\end{tabular} & 713.700 & $3541.455 \mathrm{~d}$ & 1.60 & 6.25 \\
\hline & $\mathrm{I}_{4}$ Full irrigation & 761.300 & $3599.175 \mathrm{~d}$ & 0.00 & 0.00 \\
\hline \multirow{4}{*}{ Velko } & $\begin{array}{c}\mathrm{I}_{1} \text { Stopping of } \\
\text { irrigation after } 60 \text { days }\end{array}$ & 455.370 & $4168.790 \mathrm{c}$ & 27.08 & 40.19 \\
\hline & \begin{tabular}{|c|}
$\mathrm{I}_{2}$ Stopping of \\
irrigation after 75 days
\end{tabular} & 632.770 & $4178.780 \mathrm{c}$ & 26.90 & 16.88 \\
\hline & $\begin{array}{c}\mathrm{I}_{3} \text { Stopping of } \\
\text { irrigation after } 90 \text { days }\end{array}$ & 713.700 & $5149.475 \mathrm{~b}$ & 9.92 & 6.25 \\
\hline & $\mathrm{I}_{4}$ Full irrigation & 761.300 & $5716.685 \mathrm{a}$ & 0.00 & 0.00 \\
\hline
\end{tabular}




\begin{tabular}{|c|c|c|c|c|c|}
\hline \multirow{4}{*}{ Local } & $\begin{array}{c}\mathrm{I}_{1} \text { Stopping of } \\
\text { irrigation after } 60 \text { days }\end{array}$ & 455.370 & $2915.600 \mathrm{e}$ & 20.56 & 40.19 \\
\hline & $\begin{array}{c}\mathrm{I}_{2} \text { Stopping of } \\
\text { irrigation after } 75 \text { days }\end{array}$ & 632.770 & $3389.940 \mathrm{~d}$ & 7.64 & 16.88 \\
\hline & \begin{tabular}{|c|}
$\mathrm{I}_{3}$ Stopping of \\
irrigation after 90 days
\end{tabular} & 713.700 & $3491.690 \mathrm{~d}$ & 4.86 & 6.25 \\
\hline & $\mathrm{I}_{4}$ Full irrigation & 761.300 & $3670.215 \mathrm{~d}$ & 0.00 & 0.00 \\
\hline & & & $\mathrm{LSD}_{.05} 404.034$ & $\mathrm{SE}=2.943$ & \\
\hline \multicolumn{6}{|c|}{ Qlyasan location } \\
\hline \multirow{4}{*}{$\begin{array}{l}\text { Barolo } \\
\text { RO }\end{array}$} & $\begin{array}{c}\mathrm{I}_{1} \text { Stopping of } \\
\text { irrigation after } 60 \text { days }\end{array}$ & 452.040 & $3644.685 \mathrm{~d}$ & 13.06 & 40.07 \\
\hline & $\begin{array}{c}\mathrm{I}_{2} \text { Stopping of } \\
\text { irrigation after } 75 \text { days }\end{array}$ & 583.330 & $3836.160 \mathrm{~d}$ & 8.50 & 22.66 \\
\hline & $\begin{array}{c}\mathrm{I}_{3} \text { Stopping of } \\
\text { irrigation after } 90 \text { days }\end{array}$ & 706.300 & $4116.620 \mathrm{c}$ & 1.81 & 6.36 \\
\hline & $\mathrm{I}_{4}$ Full irrigation & 754.260 & $4192.285 \mathrm{c}$ & 0.00 & 0.00 \\
\hline \multirow{4}{*}{ Velko } & $\begin{array}{c}\mathrm{I}_{1} \text { Stopping of } \\
\text { irrigation after } 60 \text { days }\end{array}$ & 452.040 & $4242.050 \mathrm{c}$ & 18.27 & 40.07 \\
\hline & \begin{tabular}{|c|}
$\mathrm{I}_{2}$ Stopping of \\
irrigation after 75 days
\end{tabular} & 583.330 & $4817.030 \mathrm{~b}$ & 7.20 & 22.66 \\
\hline & \begin{tabular}{|c|}
$\mathrm{I}_{3}$ Stopping of \\
irrigation after 90 days
\end{tabular} & 706.300 & $4910.825 \mathrm{~b}$ & 5.39 & 6.36 \\
\hline & $\mathrm{I}_{4}$ Full Irrigation & 754.260 & $5190.545 \mathrm{a}$ & 0.00 & 0.00 \\
\hline \multirow{4}{*}{ Local } & $\begin{array}{c}\mathrm{I}_{1} \text { Stopping of } \\
\text { irrigation after } 60 \text { days }\end{array}$ & 452.040 & $3283.010 \mathrm{e}$ & 10.55 & 40.07 \\
\hline & \begin{tabular}{|c|}
$\mathrm{I}_{2}$ Stopping of \\
irrigation after 75 days
\end{tabular} & 583.330 & $3214.190 \mathrm{e}$ & 12.43 & 22.66 \\
\hline & $\begin{array}{c}\mathrm{I}_{3} \text { Stopping of } \\
\text { irrigation after } 90 \text { days }\end{array}$ & 706.300 & $3186.440 \mathrm{e}$ & 13.18 & 6.36 \\
\hline & $\mathrm{I}_{4}$ Full irrigation & 754.260 & $3738.295 \mathrm{~d}$ & 0.00 & 0.00 \\
\hline & & & LSD $_{.05} 253.587$ & $\mathrm{SE}=1.783$ & \\
\hline
\end{tabular}

As shown in Table 6 and Figures 3 and 4, the crop yield response factor was estimated for sunflower at the study locations were calculated according to Doorenbos and Kassam (1979). The $\mathrm{k}_{\mathrm{y}}$ values ranged from the minimum of 0.337 for the Barolo RO genotype at the second location to a maximum of 0.827 for the Velko genotype at the first location.

Table 6. Crop response factor for different sunflower genotypes to limited irrigation

\begin{tabular}{|c|c|c|c|c|}
\hline Locations & Genotypes & $\begin{array}{c}\text { Seed yield } \\
\left(\mathrm{kg} \mathrm{h}^{-1}\right)\end{array}$ & $\begin{array}{c}\text { ETa } \\
\left(\mathbf{m}^{3} \mathbf{h a}^{-1}\right)\end{array}$ & $\mathbf{K y}$ \\
\hline \multirow{3}{*}{ Kanipanka } & Barolo RO & $3346.835 \mathrm{~b}$ & 4485.506 & 0.443 \\
\hline & Velko & $4803.433 \mathrm{a}$ & 4485.506 & 0.827 \\
\hline & Local & $3366.861 \mathrm{~b}$ & 4485.506 & 0.508 \\
\hline & & LSD .05 202.017 & & $S E=0.119$ \\
\hline \multirow{3}{*}{ Qlyasan } & Barolo RO & $3947.438 \mathrm{~b}$ & 4055.878 & 0.337 \\
\hline & Velko & $4790.113 \mathrm{a}$ & 4055.878 & 0.430 \\
\hline & Local & $3355.484 \mathrm{c}$ & 4055.878 & 0.417 \\
\hline & & LSD .05 126.794 & & $S E=0.029$ \\
\hline
\end{tabular}




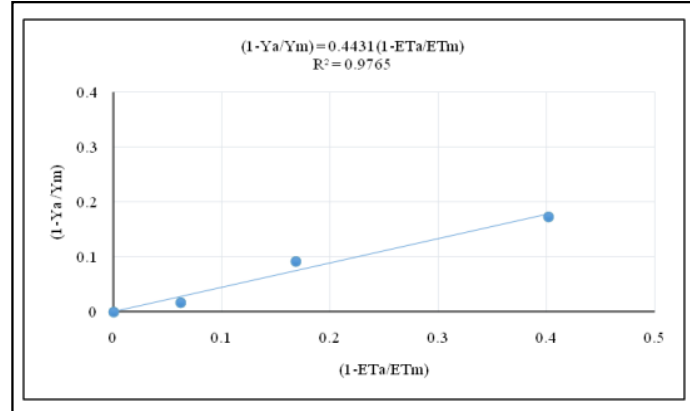

A. The relationship between yield reduction and water deficit for Sunflower Barolo RO genotype

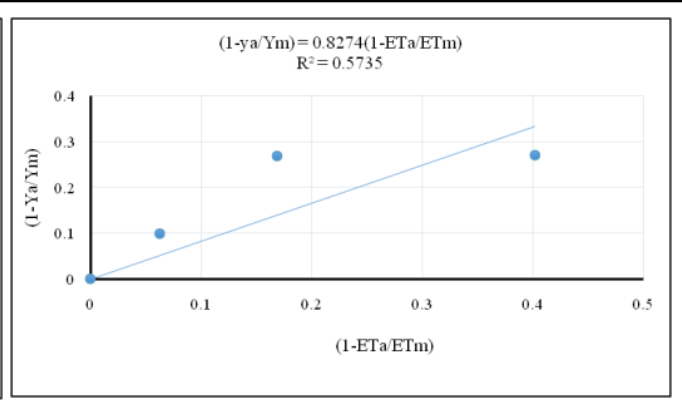

B. The relationship between yield reduction and water deficit for Sunflower Velko genotype

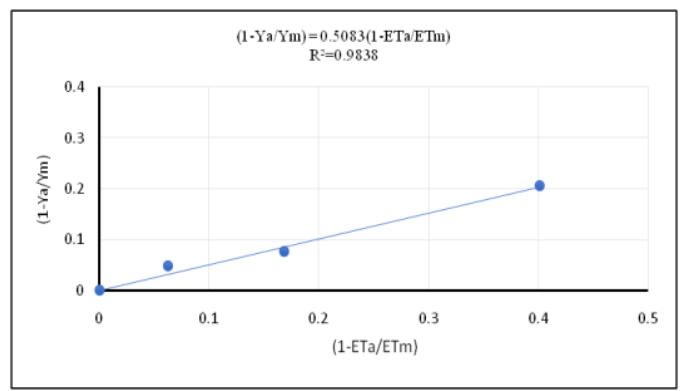

C. The relationship between yield reduction and water deficit for Sunflower Local genotype

Figure 3. The relationship between yield reduction and water deficit for sunflower genotypes at Kanipanka location

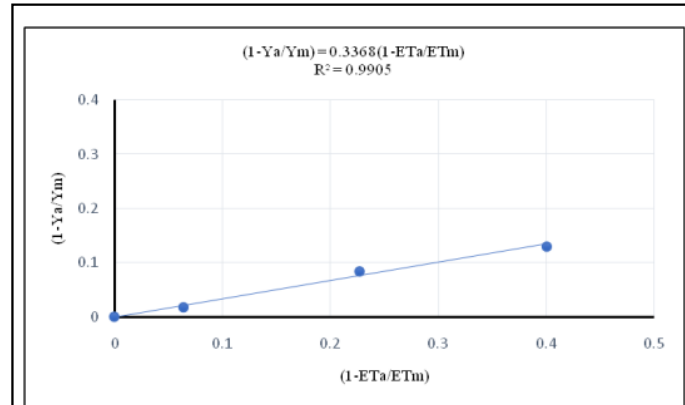

A. The relationship between yield reduction and water deficit for Sunflower Barolo RO genotype

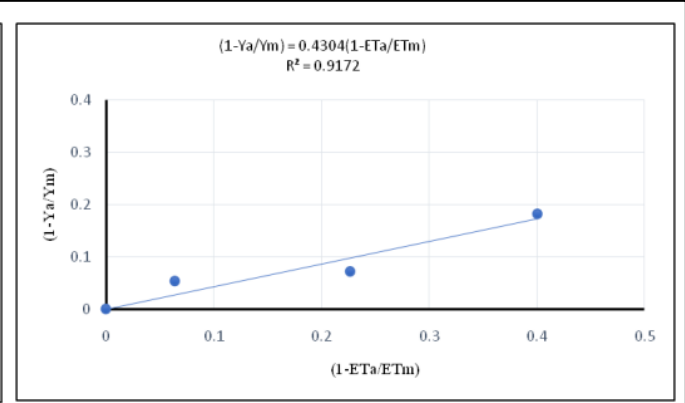

B. The relationship between yield reduction and water deficit for Sunflower Velko genotype

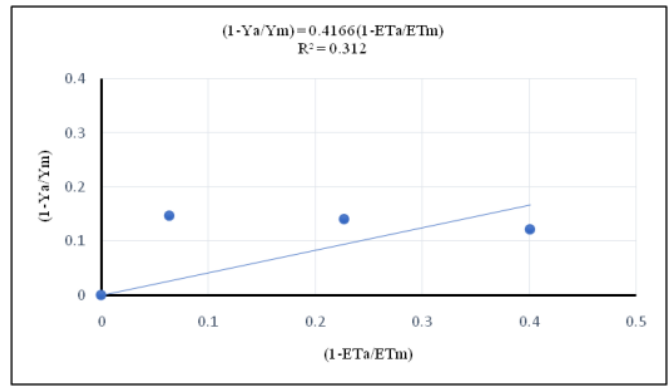

C. The relationship between yield reduction and water deficit for Sunflower Local genotype

Figure 4. The relationship between yield reduction and water deficit for sunflower genotypes at Qlyasan location 
The result displayed in Table 6 revealed that among the genotypes, Barolo RO exhibited the least value of $\mathrm{k}_{\mathrm{y}}$ at both locations. The lower result indicates that this genotype is the most proper one for the study area as a part of drought-prone environments. Further examination of Table 6, revealed that the $\mathrm{k}_{\mathrm{y}}$ values for both locations exhibited similar trends. The Barolo RO and Velko offered the minimum and maximum values for $\mathrm{k}_{\mathrm{y}}$ respectively at both locations.

Based on the $\mathrm{k}_{\mathrm{y}}$ values listed in Table 7, deficit irrigation may be needed at different stages for water limiting areas. Also, it was noticed that the uniformity coefficient values were below 0.86 . The Velko genotype showed a uniformity coefficient of 0.55 . Therefore, statistical differences between genotype and locations can exist.

Table 7. Yield response factor (Ky) of sunflower genotypes under different irrigation treatments

\begin{tabular}{|c|c|c|c|c|c|c|c|c|}
\hline \multirow{2}{*}{ Genotypes } & \multicolumn{2}{|c|}{$\underset{\left(\mathbf{m}^{3} \mathbf{h a} \mathbf{a}^{-1}\right)}{\mathbf{E T a}}$} & \multicolumn{2}{|c|}{ Ky value } & \multirow{2}{*}{ Mean } & \multirow{2}{*}{$\begin{array}{c}\text { Standard } \\
\text { deviation } \\
\text { (SD) }\end{array}$} & \multirow{2}{*}{$\begin{array}{l}\text { Uniformity } \\
\text { coefficient } \\
\text { (UC) }\end{array}$} & \multirow{2}{*}{$\begin{array}{c}\text { Coefficient } \\
\text { of variance } \\
\text { (CV) } \%\end{array}$} \\
\hline & $\begin{array}{c}\text { Kanipanka } \\
\text { location }\end{array}$ & \begin{tabular}{|l|l|} 
Qlyasan \\
location
\end{tabular} & $\begin{array}{c}\text { Kanipanka } \\
\text { location }\end{array}$ & \begin{tabular}{|l|} 
Qlyasan \\
location \\
\end{tabular} & & & & \\
\hline Barolo RO & 4485.506 & 4055.878 & 0.4 & 37 & 0.390 & 0.0752 & 0.807 & 19.28 \\
\hline Velko & 4485.506 & 4055.878 & 0.827 & 0.430 & 0.629 & 0.281 & 0.554 & 44.64 \\
\hline Local & 4485.506 & 4055.878 & 0.508 & 0.417 & 0.462 & 0.065 & 0.860 & 14.02 \\
\hline
\end{tabular}

\section{Discussion}

The results of the study indicated that the highest seed yield was obtained from the control treatment (full irrigation) and drought stress statistically decreased seed yield compared to no stress. These results collaborate (Tabatabaei et al., 2012; Dehkhoda et al., 2013; and Hussain et al., 2013). Unger (1982) found that limited irrigation water resulted in higher water use efficiency than full irrigation.

The higher IWUE and WUE values were due to limited irrigation treatments. Therefore, the increase of seed yields depends on genotypes and irrigation interval. Further, it can be reported, that with one exception, there is a steady decrease in both IWUE and WUE with an increase in the amount of applied water or plant evaporation. These changes may be due to a minimum water use efficiency of $6.754 \mathrm{~kg} \mathrm{ha}^{-1} \mathrm{~mm}^{-1}$ under $\mathrm{I}_{4}$ (full irrigation) for Barolo RO genotype, and maximum water use efficiency $14.437 \mathrm{~kg} \mathrm{ha}^{-1} \mathrm{~mm}^{-1}$ under $\mathrm{I}_{1}$ (stopping of irrigation after 60 days) for Velko genotype. However, Langeroodi et al. (2014) found that the highest water use efficiency achieved under several limited irrigation treatments for sunflower in the Islamic Republic of Iran was $7.1 \mathrm{~kg} \mathrm{ha}^{-1} \mathrm{~mm}^{-1}$. Also, Demir et al. (2006) found that the highest value was $10.19 \mathrm{~kg} \mathrm{ha}^{-1} \mathrm{~mm}^{-1}$ under (full irrigation) at Bursa, Turkey. These findings support the results of Mahender et al. (2000) and Kakar and Soomro (2001) who pointed out that the increase in seed yield of sunflower depended on genotypes and irrigation intervals. Therefore, further studies are needed to investigate the important of water use efficiency on sunflower seed yields.

In regard to the genotypes, at the second and third stages, i.e., stopping of irrigation after 75 and 90 days was the critical stages for deficit irrigation. It was proved that stopping of irrigation after these periods can minimize crop yield to a great extent. Stopping of irrigation after 60 days should be preferred due to higher IWUE and WUE 
if water resources are limited and irrigation water cost is high. Sunflower genotypes showed different responses to irrigation treatments under the conditions of the experiments.

It is interesting to note that the obtained values of the crop response factor were within the range of values documented for sunflower found in the literatures. There are numerous research reports exist on yield response of sunflower to water, a host of researchers found that the $\mathrm{k}_{\mathrm{y}}$ values were in the range of 0.80-0.95 for sunflower (Doorenbos and Kassam, 1979; Moutonnet, 2002; Demir et al., 2006). Apart from this, Mila and Ali (2016) found that the $\mathrm{k}_{\mathrm{y}}$ values were in the range of 0.25 to 0.64 for the entire growing season of sunflower. The factor $\mathrm{k}_{\mathrm{y}}$ captures the essence of the complex linkages between production and water use by sunflower in this study. With no exception, all the genotypes yielded crop response factors were of less than 1.0. The decrease $\mathrm{k}_{\mathrm{y}}$ value indicates that the grown crops are more tolerant to water deficit, and recovers partially from stress, exhibiting less than proportional reductions in yield with reduced water use. Based on the $\mathrm{k}_{\mathrm{y}}$ values deficit irrigation may be needed at different stages for water limiting area. Our result collaborates with the FAO standard as reported by Steduto et al. (2012).

The Velko genotype showed a uniformity coefficient of 0.55 . Therefore, statistical differences between genotype and locations can exist. These values vary depending on season, location and intensity of water deficit (Mila and Ali, 2016).

It is apparent from obtained results that the growth stage is most responsive to irrigation was early stage (the first 60 days), compared with other stages. Therefore irrigation during this period would ensure the least yield reduction of sunflower. These results implied that irrigation at an earlier stage was much useful to increase seed yield of sunflower genotypes rather than the middle and later irrigation. Enough root penetration without water deficit during the early stage may be responsible for the crop tolerance to drought at later stages of growth. Additionally, plant stress due to the increased production of antioxidant enzymes may also contribute to plant resistance to drought (Langeroodi et al., 2014). Therefore, tolerance of sunflower plants to drought makes sunflower more valuable under the prevailing climatic condition of the study area where the climate is characterized as semiarid due to irregular and insufficient rainfall and hot weather during vegetation period for sunflower production (Flagella et al., 2002; Reddy et al., 2003).

\section{Conclusion}

The results of this study indicated that Barolo RO genotype is the most proper one for the study area as a part of drought-prone environments. Additionally, the results indicated that full irrigation at the early stage of growth was more effective to increase the seed yield of sunflower genotypes rather than the middle and later stages of its growth. Thus some water must be ensured at this stage. Also, higher response factor indicates greater water stress. Therefore, the water supply must be applied at the vegetative and pre-flowering stage. However, this will vary with location, the intensity of water deficit, and growth stages. In view of the obtained values of water efficiency, it is recommended to give high priority to Velko genotype coupled with stopping irrigation after 60 days. 


\section{REFERENCES}

[1] Ali, M. H. (2009): Irrigation-yield response factor of winter wheat for different growth phases. - Journal of Agrometeorology 11(1): 9-14.

[2] Ali, M. H., Hoque, M. R., Hassan, A. A., Khair, A. (2007): Effects of deficit irrigation on yield, water productivity, and economic returns of wheat. - Agricultural Water Management 92(3): 151-161.

[3] Allen, R. G., Pereira, L. S., Raes, D., Smith, M. (1998): Crop EvapotranspirationGuidelines for Computing Crop Water Requirements. - FAO Irrigation and Drainage Paper 56. 300(9): D05109. FAO, Rome.

[4] Andrich, G., Zinnai, A., Balzini, S., Silvestri, S., Galoppini, C. (1996): The effect of drought stress on some characteristics of sunflower seeds. - Agricoltura Mediterranea 126: 285-291.

[5] Asraf, T., Ali, M. H. (2015): Water table dynamics and trend in three Upazilas of Rajshahi district (Barind area), Bangladesh. - Asian Acad. Res. J. Multidiscip 2: 286-310.

[6] Ayas, S., Korukçu, A. (2010): Water-yield relationships in deficit irrigated potato. Ziraat Fakültesi Dergisi, Uludağ Üniversitesi 24(2): 23-36.

[7] Beg, A., Pourdad, S. S., Alipour, S. (2007): Row and plant spacing effects on agronomic performance of sunflower in warm and semi-cold areas of Iran. - Helia 30(47): 99-104.

[8] Black, C. A., Evans, D. D., Dinauer, R. C. (1965): Methods of soil analysis. Vol. 9. American Society of Agronomy, Madison, WI, pp. 653-708.

[9] Dehkhoda, A., Naderidarbaghshahi, M., Rezaei, A., Majdnasiri, B. (2013): Effect of water deficiency stress on yield and yield component of sunflower cultivars in Isfahan. Int J Farming Allied Sci 2: 1319-1324.

[10] Demir, A. O., Göksoy, A. T., Büyükcangaz, H., Turan, Z. M., Köksal, E. S. (2006): Deficit irrigation of sunflower (Helianthus annuus L.) in a sub-humid climate. - Irrigation Science 24(4): 279-289.

[11] Devitt, D. A., Morris, R. L., Bowman, D. C. (1992): Evapotranspiration, crop coefficients, and leaching fractions of irrigated desert turfgrass systems. - Agronomy Journal 84(4): 717-723.

[12] Doorenbos, J., Kassam, A. H. (1979): Yield response to water. - Irrigation and Drainage Paper 33: 257.

[13] Duarte, J. D. L., Lima, A. D., Nascimento, R. S., Viana, T. D. A., Saraiva, K. R., de Azevedo, B. M. (2012): Water use efficiency in sunflower (Helliantusannuus L.) oil production, under water deficit. - Revista Brasileira de Agricultura Irrigada 6(3): 166175.

[14] Fereres, E., Gimenez, C., Fernandez, J. M. (1986): Genetic variability in sunflower cultivars under drought. I. Yield relationships. - Australian Journal of Agricultural Research 37(6): 573-582.

[15] Flagella, Z., Rotunno, T., Tarantino, E., Di Caterina, R., De Caro, A. (2002): Changes in seed yield and oil fatty acid composition of high oleic sunflower (Helianthus annuus L.) hybrids in relation to the sowing date and the water regime. - European Journal of Agronomy 17(3): 221-230.

[16] Gomes, E. P., Fedri, G., Ávila, M. R., Biscaro, G. A., Rezende, R. K., Jordan, R. A. (2012): Produtividade de grãos, óleo e massaseca de girassol sob diferenteslâminas de irrigaçãosuplementar. - Revista Brasileira de Engenharia Agricola e Ambiental-Agriambi 16(3).

[17] González, J., Mancuso, N., Ludueña, P. (2013): Sunflower yield and climatic variables. Helia 36(58): 69-76.

[18] Hussain, S., Saleem, M. F., Ali, A., Iqbal, J., Shakir, M. A. (2013): Yield and quality improvement of sunflower (Helianthus annuus L.) Hybrid through aba application under water deficit conditions. - The Journal of Animal Plant Sciences 23(4): 1158-1165. 
[19] Ibrahim, H. M. (2012): Response of some sunflower hybrids to different levels of plant density. - APCBEE Procedia 4: 175-182.

[20] Iqbal, N., Ashraf, M., Ashraf, M. Y., Azam, F. (2005): Effect of exogenous application of glycinebetaine on capitulum size and achene number of sunflower under water stress. Int J Biol Biotech 2: 765-771.

[21] Kakar, A. A., Soomro, A. G. (2001): Effect of water stress on the growth, yield and oil content of sunflower. - Pak. J. Agri. Sei. 38: 1-2.

[22] Kang, S., Shi, W., Zhang, J. (2000): An improved water-use efficiency for maize grown under regulated deficit irrigation. - Field Crops Research 67(3): 207-214.

[23] Karaata, H. (1991): Water-production functions of sunflower plant in Kurklareli conditions. - Ph.D. Thesis. TC Ministry of Agriculture and Rural Affairs General Directorate of Rural Services Rural Services ATATÜRK Research Institute Publications, $28,24$.

[24] Karim, T. H., Karim, K. (2001): Water demand of crops at Smaquly Watershed/Koya. FAO Representation in Iraq. FAO Coordination Office for Northern Iraq, Erbil. Iraq.

[25] Krizmanić, M., Liović, I., Mijić, A., Bilandžić, M., Krizmanić, G. (2003): Genetic potential of OS sunflower hybrids in different agroecological conditions. - Sjemenarstvo 20(5/6): 237-245.

[26] Kurdistan Regional Government (2018): Kurdistan's geography and climate. http://cabinet.gov.krd/a/d.aspx?s=010000\&l=12\&a=18656.

[27] Langeroodi, A. R. S., Kamkar, B., da Silva, J. A. T., Ataei, M. (2014): Response of sunflower cultivars to deficit irrigation. - Helia 37(60): 37-58.

[28] Lorenz, O. A., Maynard, D. N. (1980): Knott's handbook for vegetable growers. - John Wiley \& Sons, New York.

[29] Mahender, S., Harbir, S., Tej, S., Jhorar, R. K., Singh, B. P. (2000): Seed yield, water use and water-use efficiency of sunflower (Helianthus annuus) genotypes under irrigation and nitrogen variables. - Indian Journal of Agronomy 45(1): 188-192.

[30] Mancosu, N., Snyder, R. L., Kyriakakis, G., Spano, D. (2015): Water scarcity and future challenges for food production. - Water 7(3): 975-992.

[31] Mila, A. J., Ali, M. H. (2016): Irrigation yield response factor of mustard at different growth phases. - International Journal of Experimental Agriculture 6(1): 15-21.

[32] Moutonnet, P. (2002): Yield Response Factors of Field Crops to Deficit Irrigation. Water Reports 22, Deficit Irrigation Practices. FAO, Rome

[33] Orgaz, F., Mateos, L., Fereres, E. (1992): Season length and cultivar determine the optimum evapotranspiration deficit in cotton. - Agronomy Journal 84(4): 700-706.

[34] Reddy, G. K. M., Dangi, K. S., Kumar, S. S., Reddy, A. V. (2003): Effect of moisture stress on seed yield and quality in sunflower. - Journal of Oilseeds Research 20: 282-283.

[35] Rockström, J., Falkenmark, M., Karlberg, L., Hoff, H., Rost, S., Gerten, D. (2009): Future water availability for global food production: the potential of green water for increasing resilience to global change. - Water Resources Research 45(7).

[36] Rosegrant, M. W., Cai, X., Cline, S. A. (2002): World Water and Food to 2025: Dealing with Scarcity. - Intl Food Policy Res Inst., Washington, DC.

[37] Sarkar, A. A., Ali, M. H. (2009): Water table dynamics of Dhaka city and its long-term trend analysis using the "MAKE SENSE" model. - Water International 34(3): 373-382.

[38] Steduto, P., Hsiao, T. C., Fereres, E., Raes, D. (2012): Crop Yield Response to Water (Vol. 1028). - FAO, Rome.

[39] Tabatabaei, S. A., Rafiee, V., Shakeri, E., Salmani, M. (2012): Responses of sunflower (Helianthus annuus L.) to deficit irrigation at different growth stages. - International Journal of Agriculture: Research and Review 2(5): 624-629.

[40] Thomas, G. W. (1996): Soil pH and Soil Acidity. - In: Sparks, D. L. (eds.) Methods of Soil Analysis, Part 3 - Chemical Methods. SSSA, ASA, Madison, WI, pp. 475-490.

[41] Unger, P. W. (1982): Time and frequency of irrigation effects on sunflower production and water use. - Soil Science Society of America Journal 46(5): 1072-1076. 
[42] Vörösmarty, C. J., Green, P., Salisbury, J., Lammers, R. B. (2000): Global water resources: vulnerability from climate change and population growth. - Science 289(5477): 284-288.

[43] WWP (2017): The United Nations World Water Development Report 2017. Wastewater: The Untapped Resource. - UN, New York.

[44] XLSTAT (2017): Data Analysis and Statistical Solution for Microsoft Excel. Addinsoft, Paris, France.

\section{APPENDIX}

Appendix 1. Mean squares of variance analysis for seed yield and some irrigation treatments at both locations

\begin{tabular}{|c|c|c|c|c|}
\hline S.O.V & d.f & $\begin{array}{l}\text { Seed yield } \\
\left(\mathrm{kg} \mathrm{ha}^{-1)}\right.\end{array}$ & $\begin{array}{c}\text { Irrigation water use } \\
\begin{array}{c}\text { efficiency } \\
\left(\mathrm{kg} \mathrm{ha}^{-1} \mathbf{~ m m}^{-1}\right)\end{array}\end{array}$ & $\begin{array}{c}\text { Water use } \\
\text { efficiency } \\
\left(\mathrm{kg} \mathrm{ha}^{-1} \mathrm{~mm}^{-1}\right)\end{array}$ \\
\hline \multicolumn{5}{|c|}{ Kanipanka location } \\
\hline Block & 2 & 112716.4 & 0.381 & 0.778 \\
\hline Irrigation & 3 & $1726819^{* *}$ & $6.275^{\text {** }}$ & $12.807^{* *}$ \\
\hline $\mathrm{E}(\mathrm{a})$ & 6 & 49336.73 & 0.189 & 0.386 \\
\hline Genotype & 2 & $8371629^{* *}$ & $20.472^{* *}$ & $41.780^{* *}$ \\
\hline Irrigation $\times$ genotype & 6 & $287980^{\text {*** }}$ & $0.433^{*}$ & $0.884^{*}$ \\
\hline $\mathrm{E}(\mathrm{b})$ & 16 & 54482.28 & 0.158 & 0.323 \\
\hline \multicolumn{5}{|c|}{ Qlyasan location } \\
\hline Block & 2 & 90154.22 & 0.217 & 0.513 \\
\hline Irrigation & 3 & $658319.5^{\text {** }}$ & $12.131^{\text {** }}$ & $28.713^{* *}$ \\
\hline $\mathrm{E}(\mathrm{a})$ & 6 & 42885.49 & 0.102 & 0.24 \\
\hline Genotype & 2 & $6237340^{* * *}$ & $16.168^{\text {** }}$ & $38.269^{\text {*** }}$ \\
\hline Irrigation $\times$ genotype & 6 & $105323.3^{\text {** }}$ & $0.141^{*}$ & $0.334^{*}$ \\
\hline$E(b)$ & 16 & 21462.21 & 0.047 & 0.111 \\
\hline
\end{tabular}

"Significant at 0.05

* Significant at 0.01 